\title{
Original
}

\section{Small Interfering RNA Targeting CD81 Decreased the Expression of Tumor Necrosis Factor-alpha and Synoviolin in SW982 Cells}

\author{
Takae Hanada $^{1)}$, Erika Fujimoto ${ }^{2)}$, Hiroki Mori ${ }^{3)}$, Toshitaka Ohashi ${ }^{4)}$, \\ Yoshifumi Ninomiya ${ }^{4)}$ and Tohru Nakanishi ${ }^{5)}$
}

\author{
${ }^{1)}$ Support Office, Shujitsu University School of Pharmacy, Okayama, Japan \\ ${ }^{2)}$ Kyokuto Hospital, Okayama, Japan \\ ${ }^{3)}$ Faculty of Education, Shujitsu University, Okayama, Japan \\ ${ }^{4)}$ Okayama University Graduate School of Medicine, Dentistry and Pharmaceutical Sciences, Okayama, Japan \\ ${ }^{5)}$ Molecular Biology and Clinical Diagnosis, Shujitsu University Graduate School of Pharmacy, Okayama, Japan \\ (Accepted for publication, February 13, 2013)
}

\begin{abstract}
Rheumatoid arthritis (RA), one of the most common articular diseases, is characterized by chronic inflammation, hyperproliferation of synovial cells, and bone destruction. CD81, which belongs to a family of cell-surface proteins (tetraspanin), is known to be up-regulated in RA synoviocytes. Recently, we showed the therapeutic effect of using small interfering RNA in targeting CD81 (CD81 siRNA) for RA. Here we show how tumor necrosis factor-alpha (TNF- $\alpha$ ) can induce the expression of synoviolin, which is decreased by the addition of CD81 siRNA in SW982 cells. In addition, CD81 siRNA decreases the expression of TNF- $\alpha$ in SW982 cells, suggesting that TNF- $\alpha$ stimulates the expression of synoviolin via CD81. Furthermore, knock down of CD81 by the addition of CD81 siRNA decreases the expression of TNF- $\alpha$, and TNF- $\alpha$-induces expression of synoviolin in direct and indirect ways. These results in SW982 cells might explain the therapeutic effect of CD81 siRNA in animal models.
\end{abstract}

Key words: Rheumatoid arthritis, CD81, Synoviolin, Tumor necrosis factor-alpha, SW982 cell, siRNA

\section{Introduction}

Rheumatoid arthritis (RA) is one of the most common articular diseases, characterized by chronic inflammation, hyperproliferation of synovial cells, and bone destruction. It is known that inflammatory cytokines, such as tumor necrosis factoralpha (TNF- $\alpha$ ) and interleukin-1beta (IL-1 $\beta$ ), are associated with the pathological processes of RA. In particular, TNF- $\alpha$ plays a pivotal role in RA through the accumulation of inflammatory cells and the perpetuation of inflammation, leading to bone destruction. Infliximab, an anti-TNF- $\alpha$ chimeric monoclonal antibody, was marketed in Japan in 2003 as a therapeutic agent for RA. However, it is reported that TNF- $\alpha$ blockade therapy rarely leads to complete remission of RA, and nearly $30 \%$ of RA patients do not respond to therapy at all ${ }^{1)}$. Recently, it was revealed that nonresponders to infliximab therapy had higher synoviolin, a novel E3 ubiquitin ligase, expression in peripheral blood and synoviocytes compared with those who responded to infliximab ${ }^{2}$. It is well known that E3 ubiquitin ligases are crucial in the endoplasmic reticulum (ER)

Correspondence to: Dr. Tohru Nakanishi, Shujitsu University Graduate School of Pharmacy, 1-6-1 Nishigawara, Okayama, 7038516 Japan; Tel: +81-86-271-8379; Fax: +81-86-271-8320; Email: torhoshi@shujitsu.ac.jp -associated degradation (ERAD) system, a process that is indispensable for elimination of unfolded proteins in the ER and that accompanies apoptosis. Synoviolin has been identified as a rheumatoid regulator. Recent studies demonstrated that synoviolin functions as a rheumatoid regulator for the overgrowth of synovial tissues in patients with RA. Mice with overexpression of synoviolin developed spontaneous arthropathy that was accompanied by synovial dysplasia. Conversely, synoviolin/hrd $1^{+/-}$ mice were resistant to collagen-induced arthritis (CIA) via enhanced apoptosis of synovial cells. Thus, synoviolin expression appears to be highly associated with the development of RA through apoptosis ${ }^{3-5)}$.

Our previous study on gene profiling of RA synoviocytes using genome-wide DNA chips found that CD81 increased in RA synoviocytes compared with osteoarthritis (OA) synoviocytes ${ }^{6,7)}$, and a CIA rat model treated with small interfering RNA targeting CD81 (CD81 siRNA) showed that CD81 siRNA decreased the expression of synoviolin ${ }^{8,9}$. OA is not associated with abnormalities of synoviocytes, whereas RA is associated with an abnormality in these cells. Thus, we started to analyze the relationship between synoviolin expression and the activation of 
A

\begin{tabular}{|l|l|}
\hline & Sequence \\
\hline \multirow{2}{*}{ siHsCD81-1 } & 5'-rCrGrCrCUrGUrGUrAUrAArArCrGUUUrC-3' \\
\cline { 2 - 2 } & 5'-rArArCrGUUrAUrAUrArCrArCrArGrGrCrGrGU-3' \\
\hline \multirow{2}{*}{ siHsCD81-2 } & 5'-rCUrCUrGrCUrArCrArCrGUrArGrCrCUUUU-3' \\
\cline { 2 - 2 } & 5'-ArArGrGrCUrArCrGUrGUrArGrCrArGrArGUrA-3' \\
\hline \multirow{2}{*}{ siHsCD81-3 } & 5'-rCrGrGArArCrArGrCUrCrCrGUrGUrArCUrGrA-3' \\
\cline { 2 - 2 } & 5'-rArGUrArCrArCrGrGrArGrCUrGrCUrGUUrCrCrGrGrA-3' \\
\hline
\end{tabular}

B

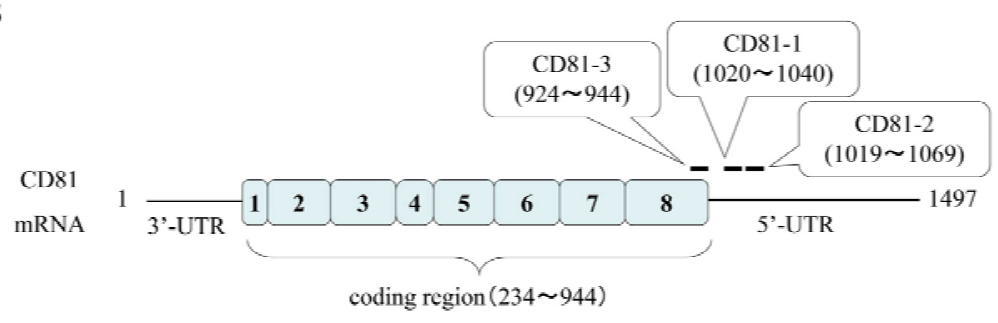

Figure 1. Construction of CD81 siRNA. A. Nucleotide sequence of CD81 siRNA. B. Binding sites of CD81 siRNA

CD81. We suggest here that CD81 knock down (KD) by siRNA in SW982 cells does not decrease the resting expression of synoviolin, but it decreases TNF- $\alpha$-induced expression of synoviolin and TNF- $\alpha$ expression itself.

There are many studies about RA, but the exact pathogenesis of RA cannot be explained. In this study, we showed the relationship between synoviolin, CD81, and inflammatory cytokines, especially TNF- $\alpha$, in RA.

\section{Materials and Methods}

\section{Cell culture}

SW982, a human synovial sarcoma cell line, was maintained in Dulbecco's modified Eagle's medium (DMEM) with low glucose (Invitrogen, Carlsbad, CA) supplemented with $10 \%$ fetal calf serum in a humidified atmosphere containing $5 \% \mathrm{CO}_{2}$ at $37^{\circ} \mathrm{C}$. Cells were seeded at a density of $0.4 \times 10^{5}$ cells/well or $0.5 \times 10^{5}$ cells/well in 24 well-plates. Cells were routinely subcultured twice a week. Cells were used after two passages. Construction of three CD81 siRNA were shown in Figure 1.

As shown in Figure 2A, SW982 cells were seeded at a density of $0.5 \times 10^{5}$ cells/well. After $24 \mathrm{~h}$ incubation, the cells were treated with siRNA for $24 \mathrm{~h}$. After that, quantitative real-time polymerase chain reaction (PCR) was performed. The transfection of siRNA into SW982 cells was performed using TransIT-TKO ${ }^{\circledR}$ Transfection Reagent (Mirus Bio, Madison, WI) or FuGENE 6 Transfection Reagent (Roche Applied Science, Indianapolis, IN) at the concentrations described in Fig. 2.

As shown in Figure 2B, SW982 cells were seeded at a density of $0.5 \times 10^{5}$ cells/well. When SW982 cells were seeded, siRNA was co-administered at a final concentration of $10 \mathrm{nM}$. After $24 \mathrm{~h}$ incubation, quantitative real-time PCR was performed. The transfection of siRNA into SW982 cells was performed using TransIT-TKO ${ }^{\circledR}$ Transfection Reagent at the concentration described in Fig. 2 (total $54 \mu \mathrm{l}$ ).

As shown in Figure 2C, SW982 cells were seeded at a density of $0.5 \times 10^{5}$ cells/well. When SW982 cells were seeded, siRNA was co-administered at a final concentration of $10 \mathrm{nM}$. After $24 \mathrm{~h}$ incubation, quantitative real-time PCR was performed. The transfection of siRNA into SW982 cells was performed using TransIT-TKO Transfection Reagent (Reagent:Opti-MEM $=4 \mu 1$ : $50 \mu 1)$.

As shown in Figure 3, SW982 cells were seeded at a density of $0.4 \times 10^{5}$ cells/well. Then, TNF- $\alpha$ (recombinant human TNF- $\alpha$ / TNFSFIA (R\&D Systems, Minneapolis, MN) was co-administered at a final concentration of $20 \mathrm{ng} / \mathrm{ml}$. After 24 or $48 \mathrm{~h}$, quantitative real-time PCR was performed.

As shown in Figure 4, SW982 cells were seeded at a density of $0.5 \times 10^{5}$ cells/well. Then, siRNA was co-administered at final concentration of $10 \mathrm{nM}$. After 24,48 , or $72 \mathrm{~h}$, quantitative realtime PCR was performed.

As shown in Figure 5, SW982 cells were seeded at density of $0.5 \times 10^{5}$ cells/well. Then, siRNA and TNF- $\alpha$ were co-administered at a final concentration of $10 \mathrm{nM}$ and $20 \mathrm{ng} / \mathrm{ml}$. After $48 \mathrm{~h}$, quantitative real-time PCR was performed.

As shown in Figure 6, SW982 cells were seeded at a density of $0.5 \times 10^{5}$ cells/well. Then, siRNA was co-administered at a final concentration of $10 \mathrm{nM}$. After $48 \mathrm{~h}$, quantitative real-time PCR was performed.

\section{RNA interference}


Takae Hanada et al.: CD81 siRNA decreased the Expression of TNF- $\alpha$

A

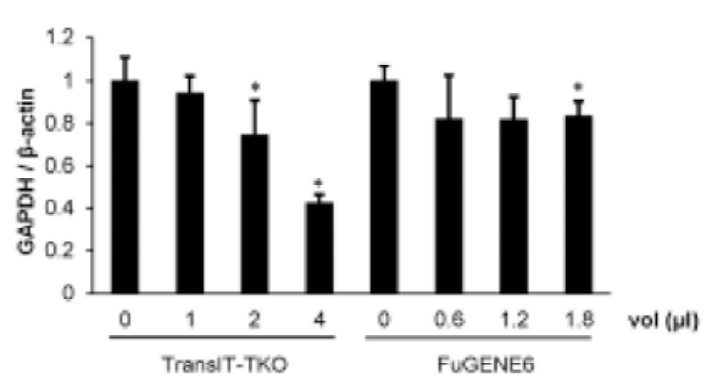

B

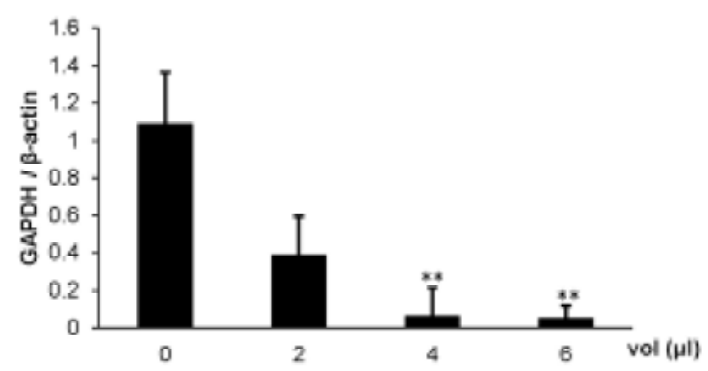

$\mathrm{C}$

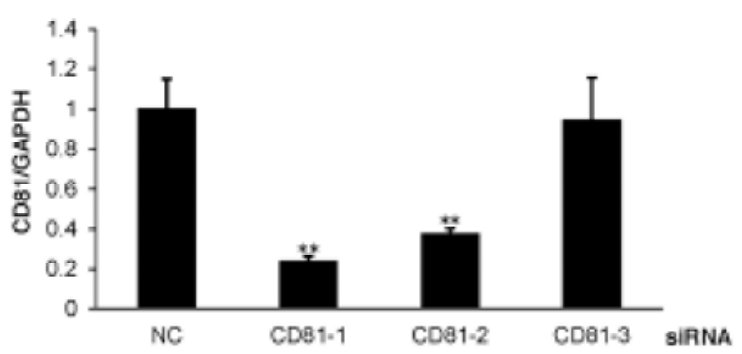

Figure 2. Establishment of the method of siRNA interference in SW982 cells

A.Examination of transfection reagents

SW982 cells were seeded at a density of $0.5 \times 10^{5}$ cells/well. After $24 \mathrm{~h}$ incubation, the cells were treated with siRNA for $24 \mathrm{~h}$. After that, quantitative real-time PCR was performed. The transfection of siRNA into SW982 cells was performed using TransIT-TKO ${ }^{\circledR}$ Transfection Reagent or FuGENE 6 Transfection Reagent at the concentrations described in Fig.2.

B.Examination of the volume of the transfection reagent

SW982 cells were seeded at density of $0.5 \times 10^{5}$ cells/well. When SW982 cells were seeded, siRNA was co-administered at a final concentration of $10 \mathrm{nM}$. After $24 \mathrm{~h}$ incubation, quantitative real-time PCR was performed. The transfection of siRNA into SW982 cells was performed using TransIT-TKO ${ }^{\circledR}$ Transfection Reagent at the concentration described in Fig. 2.

C.Examination of siRNA

SW982 cells were seeded at a density of $0.5 \times 10^{5}$ cells/well. When SW982 cells were seeded, siRNA was co-administered at a final concentration of $10 \mathrm{nM}$. After $24 \mathrm{~h}$ incubation, quantitative real-time PCR was performed. The transfection of siRNA into SW982 cells was performed using TransIT-TKO ${ }^{\circledR}$.

NC: negative control siRNA; ${ }^{*} \mathrm{p}<0.05 ;{ }^{* *} \mathrm{p}<0.01$; error bar, ${ }^{ \pm} \mathrm{SD}$ ( $\mathrm{n}=2$ or 3)

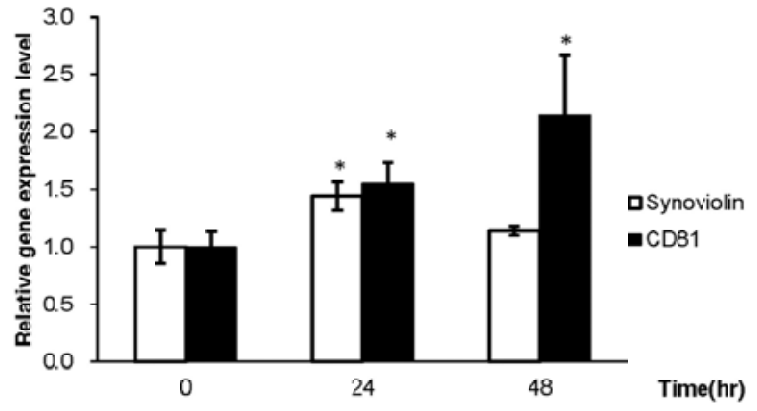

Figure 3. TNF- $\alpha$ stimulation effects on synoviolin and CD81 expression in SW982 cells

SW982 cells were seeded at a density of $0.4 \times 10^{5}$ cells $/$ well. Then, TNF- $\alpha$ was co-administered at a final concentration of $20 \mathrm{ng} / \mathrm{ml}$. After 24 or $48 \mathrm{~h}$, quantitative real-time PCR was performed. * $\mathrm{p}<0.05$; error bar: \pm SD $(n=3)$

CD81 RNA interference (RNAi) sequences were determined using the design software of Enhanced siDirect (http://sidirect.jp/ esd/modules/modsidirect/), and three CD81 small interfering RNA duplexes (siRNA) were synthesized (Sigma Genosys siRNA Service, Baltimore, MD) (Fig. 1). We used BannoNegaCon (Sigma Genosys siRNA Service) as a negative control and GAPDH_Human and LaminA/C Human as positive controls (Sigma Genosys siRNA Service). Transfection of siRNA into SW982 cells was performed using TransIT-TKO Transfection Reagent or FuGENE 6 Transfection Reagent.

\section{Quantitative real-time RT-PCR}

Total RNA was extracted from cells and was reverse transcribed using a Prime Script RT reagent kit (Takara Bio, Shiga, Japan) according to the manufacturer's instructions. Quantitative PCR amplification was performed using Premix Ex Taq (Takara Bio) or THUNDERBIRD Probe qPCR Mix (TOYOBO, Osaka, Japan) using Insogen (Nippon Gene, Tokyo, Japan) or RNAiso Plus (Takara Bio) and the following gene-specific primers or probes: 4326317E (Pre-Developed TaqMan ${ }^{\circledR}$ Assay Reagents, Applied Biosystems, Carlsbad, California) for human GAPDH, 4326315E (Pre-Developed TaqMan ${ }^{\circledR}$ Assay Reagents, Applied Biosystems) for human $\beta$-actin, Hs00174717_m1 (TaqMan ${ }^{\circledR}$ Gene Expression Assays, Applied Biosystems) for human CD81, Hs00174128_m1 (TaqMan ${ }^{\circledR}$ Gene Expression Assays, Applied Biosystems) for human TNF- $\alpha$, and Hs00174097_m1 (TaqMan ${ }^{\circledR}$ Gene Expression Assays) for human IL-1 $\beta$. Each reaction was run on an Applied Biosystems Prism 7000 Real-Time PCR system under the following conditions: Premix Ex Taq, $95^{\circ} \mathrm{C}$ for $10 \mathrm{~min}$ for 1 cycle, $95^{\circ} \mathrm{C}$ for $5 \mathrm{~min}$, and $60^{\circ} \mathrm{C}$ for $31 \mathrm{~min}$ for 40 cycles; and THUNDERBIRD Probe qPCR Mix, $95^{\circ} \mathrm{C}$ for $10 \mathrm{~min}$ for $1 \mathrm{cycle}$, $95^{\circ} \mathrm{C}$ for $5 \mathrm{~min}$, and $60^{\circ} \mathrm{C}$ for $31 \mathrm{~min}$ for 40 cycles. mRNA levels were normalized to GAPDH mRNA or $\beta$-actin mRNA and are presented as a ratio of mRNA expression to GAPDH mRNA or $\beta$ actin mRNA. 


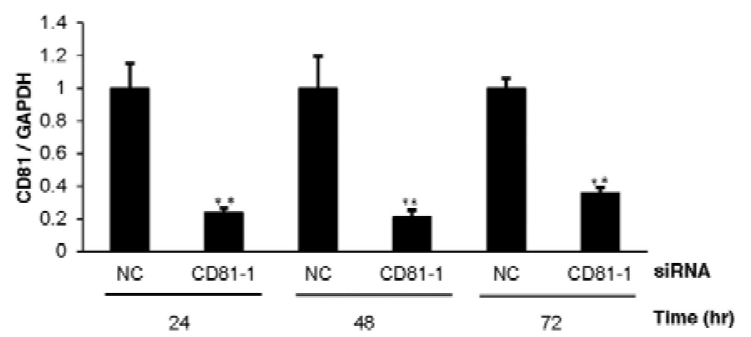

B

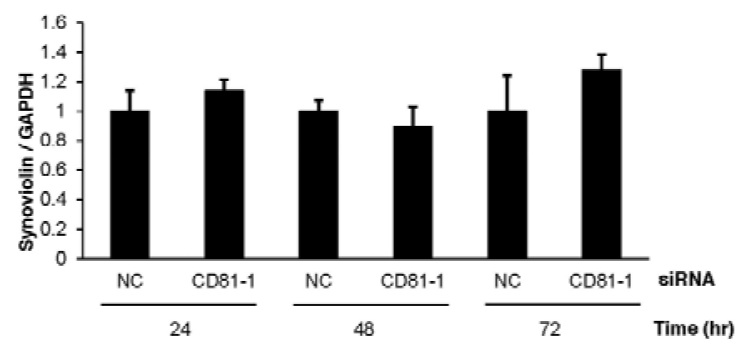

Figure 4. CD81 KD effects on synoviolin expression in SW982 cells A. Effect of siRNA on CD81 expression

SW982 cells were seeded at a density of $0.5 \times 10^{5}$ cells/well. Then, siRNA was co-administered at a final concentration of $10 \mathrm{nM}$. After 24,48 , or $72 \mathrm{~h}$, quantitative real-time PCR was performed. NC: negative control siRNA; $* * \mathrm{p}<0.01$; error bar: $\pm \mathrm{SD}(\mathrm{n}=3)$

B. Effect of siRNA on synoviolin expression

SW982 cells were seeded at a density of $0.5 \times 10^{5}$ cells/well. Then, siRNA was co-administered at a final concentration of $10 \mathrm{nM}$. After 24,48 , or $72 \mathrm{~h}$, quantitative real-time PCR was performed. NC: negative control siRNA; $* * \mathrm{p}<0.01$; error bar: $\pm \mathrm{SD}(\mathrm{n}=3)$

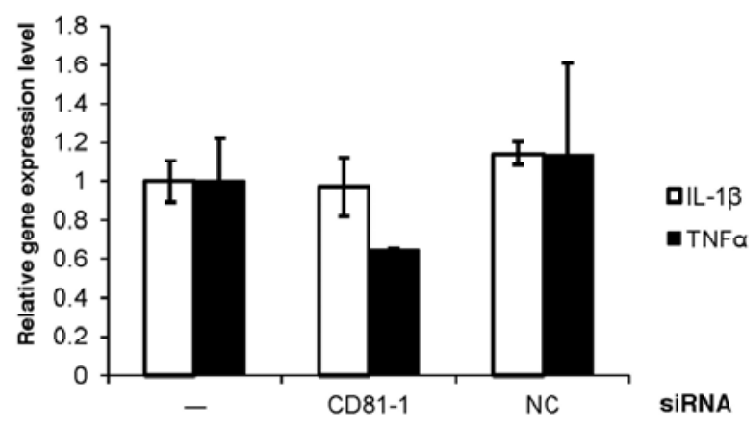

Figure 6. CD81 KD effects on TNF- $\alpha$ or IL-1 $\beta$ expression in SW982 cells

SW982 cells were seeded at a density of $0.5 \times 10^{5}$ cells/well. Then, siRNA were co-administered at a final concentration of $10 \mathrm{nM}$. After $48 \mathrm{~h}$, quantitative real-time PCR was performed. NC: negative control siRNA; error bar: $\pm \mathrm{SD}$ ( $\mathrm{n}=2$ or 3 ).

\section{Results}

\section{Establishment of the method of siRNA interference}

We previously suggested that CD81 KD decreased synoviolin expression and suppress of arthritis in the CIA rat model. To reveal the effect of down-regulation of CD81 expression on synoviolin expression, we investigated CD81 KD effects on synoviolin

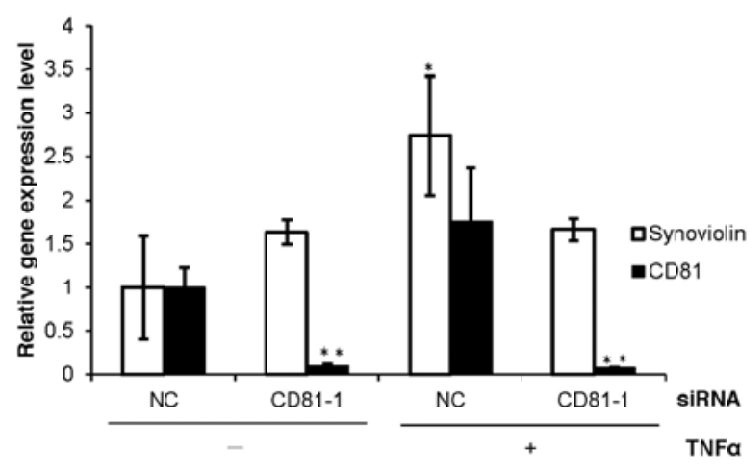

Figure 5. CD81 KD effects on synoviolin expression under the stimulation of TNF- $\pm \square$ in SW982 cells

SW982 cells were seeded at a density of $0.5 \times 10^{5}$ cells/well. Then, siRNA and TNF-alpha were co-administered at a final concentration of $10 \mathrm{nM}$ and $20 \mathrm{ng} / \mathrm{ml}$, respectively. After $48 \mathrm{~h}$, quantitative realtime PCR was performed. NC: negative control siRNA; * $\mathrm{p}<0.05$; $* * \mathrm{p}<0.01$; error bar: $\pm \mathrm{SD}(\mathrm{n}=3)$

expression in SW982 cells. Before the investigation, we established the method of siRNA interference.

We compared the TransIT-TKO Transfection Reagent and the FuGENE 6 Transfection Reagent transfection efficiency using GAPDH siRNA. Using FuGENE 6 Transfection Reagent, GAPDH expression was suppressed $20 \%$ at any of the concentrations used. Using TransIT-TKO Transfection Reagent, GAPDH expression was suppressed in a dose-dependent manner, and was suppressed about $60 \%$ at a concentration of $4 \mu 1$ (Fig. 2A). After this experiment, we performed transfection of siRNA using the TransIT-TKO Transfection Reagent. Next, we compared a forward transfection method (Fig. 2A) and a reverse transfection method (Fig. 2B) using TransIT-TKO Transfection Reagent. Using the reverse transfection method, GAPDH expression was suppressed in a dose-dependent manner and was suppressed under $90 \%$ at a volume of $4 \mu \mathrm{l}$ and $6 \mu \mathrm{l}$ (Fig. 2B). After this experiment, we performed transfection of siRNA under a reverse transfection method using TransIT-TKO Transfection Reagent ( $4 \mu \mathrm{l})$. Next, we compared knock down (KD) efficiency of three types of CD81 siRNA. CD81 expression was suppressed about $80 \%$ using CD81 siRNA-1 and about $60 \%$ using CD81 siRNA-2; CD81 expression was not suppressed using CD81 siRNA-3 (Fig. 2C). After this experiment, we performed transfection of siRNA using CD81 siRNA-1.

\section{TNF- $\alpha$ stimulation effects on synoviolin and CD81 expression}

We investigated whether synoviolin and CD81 synthesis could be induced by TNF- $\alpha$ in SW982 cells. As shown in Fig. 3, TNF$\alpha$ stimulated synoviolin expression 1.5 -fold in SW982 cells after $24 \mathrm{~h}$ of stimulation, and the expression decreased to control levels after 48 h of stimulation. The expression of CD81 in SW982 cells was also stimulated with TNF- $\alpha$ by 1.5 -fold after $24 \mathrm{~h}$ of stimulation and by 2 -fold after $48 \mathrm{~h}$ of stimulation (Fig. 3 ). 
Takae Hanada et al.: CD81 siRNA decreased the Expression of TNF- $\alpha$

\section{CD81 KD effects on synoviolin expression}

Using an established method of siRNA interference, we examined CD81 KD effects on synoviolin expression. First, we confirmed the effect of CD81 siRNA-1 on the expression of CD81 in SW982 cells. Compared with the negative control (NC) siRNA, CD81 siRNA-1 suppressed CD81 expression, about 80\% after 24 or $48 \mathrm{~h}$ of transfection (Fig. 4A). In this condition, synoviolin expression was not affected by the addition of CD81 siRNA-1 in resting (not stimulated) SW982 cells (Fig. 4B). After $72 \mathrm{~h}$ of siRNA transfection, more than $90 \%$ cells were dead, so RNA extraction was performed within $48 \mathrm{~h}$ after transfection in the following experiments.

\section{CD81 KD effects on synoviolin expression under stimulation of TNF- $\alpha$}

We showed that TNF- $\alpha$ stimulated the expression of CD81 and synoviolin, especially CD81 in SW982 cells (Fig. 3). Next, we investigated the KD effect of CD81 siRNA-1 on synoviolin expression under stimulation of TNF- $\alpha$ to reveal the relationship between TNF- $\alpha$, CD81, and synoviolin. CD81 siRNA-1 suppressed CD81 expression approximately $90 \%$ regardless of stimulation of TNF- $\alpha$ when compared with NC siRNA. Synoviolin expression increased approximately 2.5 -fold under TNF- $\alpha$ stimulation in this case; in contrast, CD81 siRNA-1 did not show any effect on expression of synoviolin in resting SW982 cells (Fig. 4B). CD81 siRNA-1 decreased synoviolin expression to control levels and did not lead to increases even under TNF- $\alpha$ stimulation (Fig. 5). Thus, increased synoviolin expression by TNF- $\alpha$ was decreased equal to the resting control level by the addition of CD81 siRNA-1 in SW982 cells, indicating that CD81 is involved in TNF- $\alpha$-induced up-regulation pathway of synoviolin in SW982 cells.

\section{CD81 KD effects on TNF- $\alpha$ or IL-1ß expression}

We also investigated the KD effects of CD81 siRNA-1 on TNF$\alpha$ itself or IL-1 $\beta$ expression in SW982 cells. CD81 siRNA-1 effectively suppressed TNF- $\alpha$ expression approximately $40 \%$ in SW982 cells. In contrast, CD81 siRNA-1 did not show any effect on IL-1 $\beta$ expression (Fig. 6).

\section{Discussion}

TNF- $\alpha$ and IL- $1 \beta$ induce synoviolin expression through activation of Erk1/2-ETS1 signal-regulated kinase in synovial fibroblasts ${ }^{10)}$. Previously, we found that CD81 increased in RA synoviocytes compared with OA synoviocytes in gene profiling using genome-wide DNA chips ${ }^{6}$. We also showed that CD81 KD by CD81 siRNA decreased synoviolin expression and suppressed arthritis in a CIA rat model ${ }^{8)}$. These results suggest that CD81 is related to RA through overexpression of synoviolin, and TNF- $\alpha$, IL-1 $\beta$, and CD81 are the key molecules that induce overexpression of synoviolin. It is generally known that CD81 is associated with cell proliferation, which corresponds to previous results showing that TNF- $\alpha$ induces neoplasia of synoviocytes in RA ${ }^{11)}$. In this study, we investigated the function of CD81 in the pathogenesis of RA in relation to TNF- $\alpha$ and IL-1beta by CD 81 KD using CD81 siRNA. For effective application of siRNA in these experiments, we performed trial experiments to compare the efficiency of two transfection reagents and two transfection methods. In the case of SW982 cells, we selected TransIT-TKO Transfection Reagent and a reverse transfection method. SW982, a human synovial sarcoma cell line, is characterized by expression of inflammatory cytokines and is a useful tool for studying the expression and regulation of inflammatory cytokines ${ }^{12)}$. In this study, we showed that TNF- $\alpha$ stimulated SW982 cells to generate CD81 and synoviolin in SW982 cells (Fig. 3). This result suggests two possibilities: 1) TNF- $\alpha$ regulates synoviolin expression through CD81; or 2) TNF$\alpha$ regulates synoviolin and CD81 via separate pathways. On the other hand, CD81 siRNA decreased not resting but TNF- $\alpha$-induced expression of synoviolin (Fig. 4B and 5), and CD81 siRNA also decreased the expression of TNF- $\alpha$ itself in SW982 cells (Fig. 6). These results indicate that hypothes 1) can be selected in the case of SW982 cells and may explain the previous results that CD81 $\mathrm{KD}$ decreased synoviolin expression in vivo ${ }^{8,9}$. In the joints of RA, TNF- $\alpha$ is derived from inflammatory lymphocytes and from abnormal synoviocytes, which stimulate the expression of CD81 as well as the expression of synoviolin through CD81. On the other hand, induced CD81 stimulates the expression of TNF- $\alpha$ in synoviocytes, then leads to abnormal growth of synoviocytes and significant RA. In other words, CD81 regulates both key factors of RA, synoviolin and synoviocytes, in the up-stream, indicating that KD of CD81 may be one effective way to treat RA. Although further detailed analysis of these mechanisms, especially ligand interaction on cell surface and transcriptional regulation, should be done, our results could explain the large ameliorating effects of arthritis in CIA rats by KD of CD81 using CD81 siRNA in spite of the following three differences seen between in vitro and in vivo studies. The first difference is one of study circumstances. Examination in vitro is performed only in a cell line, whereas examination in vivo is affected by various factors such as ambient cell functions, cytokines, and signals. These interactions lead to more complicated situations. The second difference reflects the difference of cell types between SW982 cells and synoviocytes. SW982 cells are generally used as a human synovial sarcoma cell line; however, SW982 cells may have some differences from RA synoviocytes. The third difference reflects the differences in frequency and period of CD81 KD treatments. In this study, we transfected siRNA once and incubated cells for less than $72 \mathrm{~h}$ because more than $90 \%$ of SW982 cells were dead after $72 \mathrm{~h}$ of siRNA transfection. On the other hand, we transfected siRNA four times every $72 \mathrm{~h}$ in an in vivo study and analyzed the effects of 
CD81 siRNA using rat articulations 28 days after injection of collagen $^{8}$.

In the current study, we showed that CD81 siRNA suppressed TNF- $\alpha$ expression and not IL- $1 \beta$ expression in SW892 cells. It is well known that inflammatory cytokines induce synoviocyte overgrowth and bone destruction by activation of osteoclasts in RA. However, detailed signal transduction pathways between synoviolin and CD81, and the participation of TNF- $\alpha$ in this pathway are not clear. There may be some difference in the transduction pathways between the TNF- $\alpha$ and IL- $1 \beta$ activating pathways, and there may also be some additional regulatory molecules for activation of IL-1 $\beta$ in SW982 cells.

\section{Acknowledgments}

We thank Dr. Yuji Arai for valuable suggestions. We also thank Ms. Narumi Nishikawa and Ms. Yasuyo Matsuda for technical assistance. This study was supported by a Grant-in-Aid for Scientific Research from the Ministry of Education, Science, Sports, and Culture to T.N.

\section{References}

1. Tanaka Y, Takeuchi T, Inoue E, Saito K, Sekiguchi N, Sato E, Nawata M, Kameda H, Iwata S, Amano K and Yamanaka H. Retrospective clinical study on the notable efficacy and related factors of infliximab therapy in a rheumatoid arthritis management group in Japan: one-year clinical outcomes (RECONFIRM-2). Mod Rheumatol 18: 146-152, 2008

2. Toh M-L, Marotte H, Blond J-L, Jhumka U, Eljaafari A, Mougin B and Miossec P. Overexpression of synoviolin in peripheral blood and synoviocytes from rheumatoid arthritis patients and continued elevation in nonresponders to infliximab treatment. Arthr Rheumat 54: 2109-2118, 2006

3. Amano T, Yamasaki S, Yagishita N, Tsuchimochi K, Shin H, Kawahara K, Aratani S, Fujita H, Lei Zhang, Ikeda R, Fujii R, Miura N, Komiya S, Nishioka K, Maruyama I, Fukamizu A and Nakajima T. Synoviolin/Hrd1, an E3 ubiquitin ligase, as a novel pathogenic factor for arthropathy. Genes Dev 17 : 2436-2449, 2003

4. Yamasaki S, Yagishita N, Tsuchimochi K, Nishioka K and
Nakajima T. Rheumatoid arthritis as a hyper-endoplasmic reticulum-associated degradation disease. Arthr Res Therap 7: 181-186, 2005

5. Kaneko $M$, Ishiguro $M$, Niinuma $Y$, Uesugi $M$ and Nomura Y. Human HRD1 protects against ER stress-induced apoptosis through ER-associated degradation. Feder Europ Biochem Soc Let 532: 147-152, 2002

6. Mori H, Nishida K, Ozaki T, Inoue H, Setsu K, Tsujigiwa H, Nagatsuka H, Gunduz $M$ and Nakanishi T. Expression of WISP-1 (ccn4), WISP-2 (ccn5) and WISP-3 (ccn6) in rheumatoid arthritic synovium evaluated by DNA microarrays. J Hard Tissue Biol 15: 89-95, 2006

7. Mori H, Nishida K, Ozaki T, Inoue H and Nakanishi T. Isolation of a mRNA preferentially expressed in synoviocytes from rheumatoid arthritis that is identical with lumican, which encodes a collagen binding, extracellular matrix protein. J Hard Tissue Biol 17: 125-130, 2008

8. Nakagawa S, Arai Y, Mori H, Matsushita Y, Kubo T and Nakanishi T. Small interfering RNA targeting CD81 ameliorated arthritis in rats. Biochem Biophys Res Commun 388: 467-472, 2009

9. Nakanishi T, Arai Y, Mori H, Nakajima T and Kubo T. Application of tetraspanin CD81 RNAi for diagnosis and therapy of rheumatoid arthritis (RA). Arthr Res Therap 14: 52,2011

10. Beixue Gao, Karen Calhoun and Deyu Fang. The proinflammatory cytokines IL-1beta and TNF-alpha induce the expression of synoviolin, an E3 ubiquitin ligase, in mouse synovial fibroblasts via the Erk1/2-ETS1 pathway. Arthr Res Therap 8: R172, 2006

11. Grimbacher B, Aicher WK, Peter HH and Eibel H. TNFalpha induces the transcription factor Egr-1, proinflammatory cytokines and cell proliferation in human skin fibroblasts and synovial lining cells. Rheumatol Int 17:185192, 1998

12. Yamazaki $\mathrm{T}$, Yokoyama $\mathrm{T}$, Akatsu $\mathrm{H}$, Tukiyama $\mathrm{T}$ and Tokiwa T. Phenotypic characterization of a human synovial sarcoma cell line, SW982, and its response to dexamethasone. In Vitro Cell Dev Biol Anim 39: 337-339, 2003 